\title{
BMJ Open Rehabilitation nursing for motor functional recovery of acute ischaemic stroke: study protocol for a randomised controlled trial
}

\author{
Jianmiao Wang (D) , ${ }^{1}$ Yuanyuan Chen, ${ }^{2}$ Yuping Zhang, ${ }^{1}$ Mei Li, ${ }^{1}$ Jingfen Jin $^{1}$
}

To cite: Wang J, Chen Y, Zhang Y, et al. Rehabilitation nursing for motor functional recovery of acute ischaemic stroke: study protocol for a randomised controlled trial. BMJ Open 2020;10:e037391. doi:10.1136/ bmjopen-2020-037391

- Prepublication history for this paper is available online. To view these files, please visit the journal online (http://dx.doi. org/10.1136/bmjopen-2020037391).

Received 31 January 2020 Revised 10 August 2020 Accepted 22 August 2020

Check for updates

(C) Author(s) (or their employer(s)) 2020. Re-use permitted under CC BY-NC. No commercial re-use. See rights and permissions. Published by BMJ.

${ }^{1}$ Nursing Department, The Second Affiliated Hospital of Zhejiang University School of Medicine, Hangzhou, Zhejiang, China

${ }^{2}$ Neurology Department, The Second Affiliated Hospital of Zhejiang University School of Medicine, Hangzhou, Zhejiang, China

Correspondence to

Prof. Jingfen Jin;

zrjzkh|@zju.edu.cn

\section{ABSTRACT}

Introduction Stroke is the second-leading cause of death and disability in the world, and patients with stroke often suffer from functional impairments and need rehabilitation. Notably, there is much evidence that rehabilitation can lead to better mortality and morbidity outcomes. The evidence for the effectiveness of rehabilitation nursing, however, is limited. Thus, this study seeks to explore whether rehabilitation nursing is not inferior to usual rehabilitation for motor functional recovery in patients with acute ischaemic stroke.

Methods and analysis We will conduct an assessorblinded parallel randomised controlled trial of patients who meet the inclusion criteria after stratification by weighted corticospinal tract lesion load. The experimental group will receive rehabilitation nursing by trained and qualified nurses (seven consecutive days, two sessions per day, 30 min each session). The control group will receive usual rehabilitation provided by therapists (seven consecutive days, two sessions per day, 30 min each session). The primary outcome measures are the Motor Assessment Scale, the Fugl-Meyer Assessment and the Action Research Arm Test. The secondary outcome measures are the modified Rankin Scale, the modified Barthel Index and the National Institute of Health Stroke Scale. Primary and secondary outcome assessment will be performed before and after the intervention, and secondary outcome be assessed at 4 and 12 weeks follow-up. We will recruit 224 patients within a period of 1218 months from a hospital in southeastern China. Ethics and dissemination The study was approved by the Human Research Ethics Committee from the corresponding author's hospital (approval Number is Ethical Review Study No. 2018 - 112). Peerreviewed journals and presentations at national and international conferences will be used to disseminate the results.

Trial registration number NCT03702452.

\section{INTRODUCTION}

According to WHO, stroke is the secondleading cause of death and disability worldwide, representing $10.2 \%$ of all deaths and $5.2 \%$ of disability-adjusted life years

\section{Strengths and limitations of this study}

- This is a non-inferiority study of rehabilitation nursing in patients with ischaemic stroke stratified by weighted corticospinal tract lesion load in an acute setting.

- The time and intensity of motor function rehabilitation in the acute phase is limited and may affect the study results.

- This is a single-site randomised controlled trial, which may limit the generalisability of the findings.

(DALYs, is generated by summing years of life lost and years lived with disability) from all sources in 2016. ${ }^{1}$ In China, stroke has been the leading cause of death, with ischaemic stroke accounting for $69.6 \%-77.8 \%$ of the total number of strokes. ${ }^{2}$ Patients who have had a stroke demonstrate various degrees of functional impairment that lead to long-term disability for about $50 \%-70 \%$ of the victims, ${ }^{3}$ which seriously affects the patients' quality of life. ${ }^{4}$

The 2015 Global Burden of Disease study shows that the DALYs of $74 \%$ of patients worldwide can benefit from rehabilitation. ${ }^{5}$ Early rehabilitation involves providing services to restore function once the patient's condition has become stable, ${ }^{6}$ a systematic review of randomised controlled trials (RCTs), found that patients had significant improvements in scores on the Barthel Index (BI) and Fugl-Meyer Assessment (FMA) after rehabilitation as compared with standard care poststroke. ${ }^{7}$ Early rehabilitation also may result in longterm benefits by decreasing the mortality rate. $^{8}$ In a population-based study firststroke survivors who received rehabilitation, whether or not they transferred to a rehabilitation ward, had a decreased 5-year mortality rate compared with the patients who did not receive rehabilitation. ${ }^{9}$ 
Motor function recovery in the acute phase is beneficial for improving the patient's structure and function of the corticospinal tract (CST).$^{10} \mathrm{CST}$ is the largest descending nerve fibre tracts, the basis of motion control and the main structure that affects the prognosis of motor function. The ability of patients to live independently depends to a large extent on the degree of injury and repair of CST. ${ }^{11}$ Our previous study also confirmed that there was a significant correlation between CST injury and motor function recovery in the acute phase. ${ }^{12}$ Feng et al showed that weighted CST lesion load (wCST-LL) in the acute phase was a predictor of motor function recovery at 3 months after stroke. ${ }^{13}$

According to the 2018 American Heart Association and the American Stroke Association guidelines, all patients after stroke should receive early rehabilitation implemented by an organised, multidisciplinary care team (level I evidence, level A recommendation). ${ }^{14}$ It is difficult, however, to obtain rehabilitation during the acute phase in stroke wards in China. Registry data revealed that only $59.4 \%$ of patients poststroke received rehabilitation assessment during hospital admission, and only $50 \%$ were assessed by a rehabilitation therapist. ${ }^{15}$ Stroke rehabilitation exists but is rarely identified as a priority in acute care in China. The reasons may include insufficient awareness, especially regarding early rehabilitation. ${ }^{16}$ China faces the increasing challenge of helping patients to have the best recovery potential. ${ }^{17}$ Efforts should be devoted to facilitate healthcare professionals' awareness of the advantages of rehabilitation and to enhance participation. ${ }^{18}$ Stroke recovery is heterogeneous, but the importance of early intervention and rehabilitation is supported. ${ }^{19}$ Nevertheless, a standard acute stroke rehabilitation programme may need to be customised locally with additional training and task shifting to match the resources available. ${ }^{15}$

Nurses can be the best candidates for rehabilitation delivery as they play a vital role and are responsible for patients 24 hours a day. Nursing can improve the neurological function and activities of daily living (ADLs) of patients with stroke. ${ }^{20}$ In one study, an educational training programme for nurses improved nurses' knowledge and practice in clinical care as well as ADLs and self-care among patients with stroke. ${ }^{21}$ Nurses were able to translate the development of disparate physical skills into ADLs. Nurses must make full use of this aspect of their role to build on and develop new partnerships with patients beyond the boundaries of current rehabilitation, ${ }^{22}$ and small changes to the way that nurses view and conduct rehabilitation can have a major impact.

In stroke rehabilitation in the acute setting, nurses have an essential role to play. ${ }^{23}$ For instance, they should facilitate the practice of skills learnt in therapy sessions, as guided by the therapist. ${ }^{24}$ To facilitate early recovery, advanced nursing care should include a large variety of specific nursing interventions, including early mobilisation. ${ }^{25}$ Because acute inpatient rehabilitation is the first level of the three-level rehabilitation system in China, it is feasible and essential to conduct a rehabilitation nursing programme. There is currently, however, a lack of high-quality RCTs investigating in acute stroke rehabilitation, ${ }^{26} 27$ and no recommended acute rehabilitation nursing programmes.

Thus, we have developed a modified BI (MBI) graded motor function rehabilitation nursing programme through the Delphi method, which we compare with the usual rehabilitation. The programme's training of the impaired limb emphasises the use of individualised tasks to promote frequent repetitions of task-oriented movements, as this mechanism may be related to brain function reorganisation and neural plasticity based on experience and learning. ${ }^{28}$ The key components of interventions are consistent with occupational therapy's notion that engagement in occupation is a key to health and well-being. ${ }^{29}$ The programme makes it convenient for nurses to choose rehabilitation exercise items suitable for patients.

The findings of our pilot study showed that rehabilitation nursing provided a more noticeable effect on patients' functional improvement poststroke with a wCST-LL smaller than $2 \mathrm{~mL}$ (observational results, currently unpublished). We hypothesised that patients in acute ischaemic stroke who participate in a rehabilitation nursing programme would have motor function outcomes not inferior to those of the control group, and that there is difference between groups stratified by wCST-LL (less than $2 \mathrm{~mL}$ vs $2 \mathrm{~mL}$ or greater).

\section{Objective}

The objective of this protocol is to conduct an RCT to explore whether rehabilitation nursing is not inferior to usual rehabilitation for motor functional recovery using the Motor Assessment Scale (MAS), the FMA and the Action Research Arm Test (ARAT) in patients with acute ischaemic stroke stratified by wCST-LL. The secondary aim of this study is the effect of wCST-LL on outcomes.

\section{METHODS AND ANALYSIS \\ Study design}

This is a single assessor blind parallel group, and onesite RCT of rehabilitation nursing in acute ischaemic stroke. When patients are admitted to the hospital, the wCST-LL is determined first and then patients are divided into a 'large' group (wCST-LL is greater than or equal to $2 \mathrm{~mL}$ ) or 'small' group (wCST-LL is less than $2 \mathrm{~mL}$ ). Then, patients are randomly assigned to an experimental or control group according to a computer-generated random number. The experimental group will engage in rehabilitation nursing 


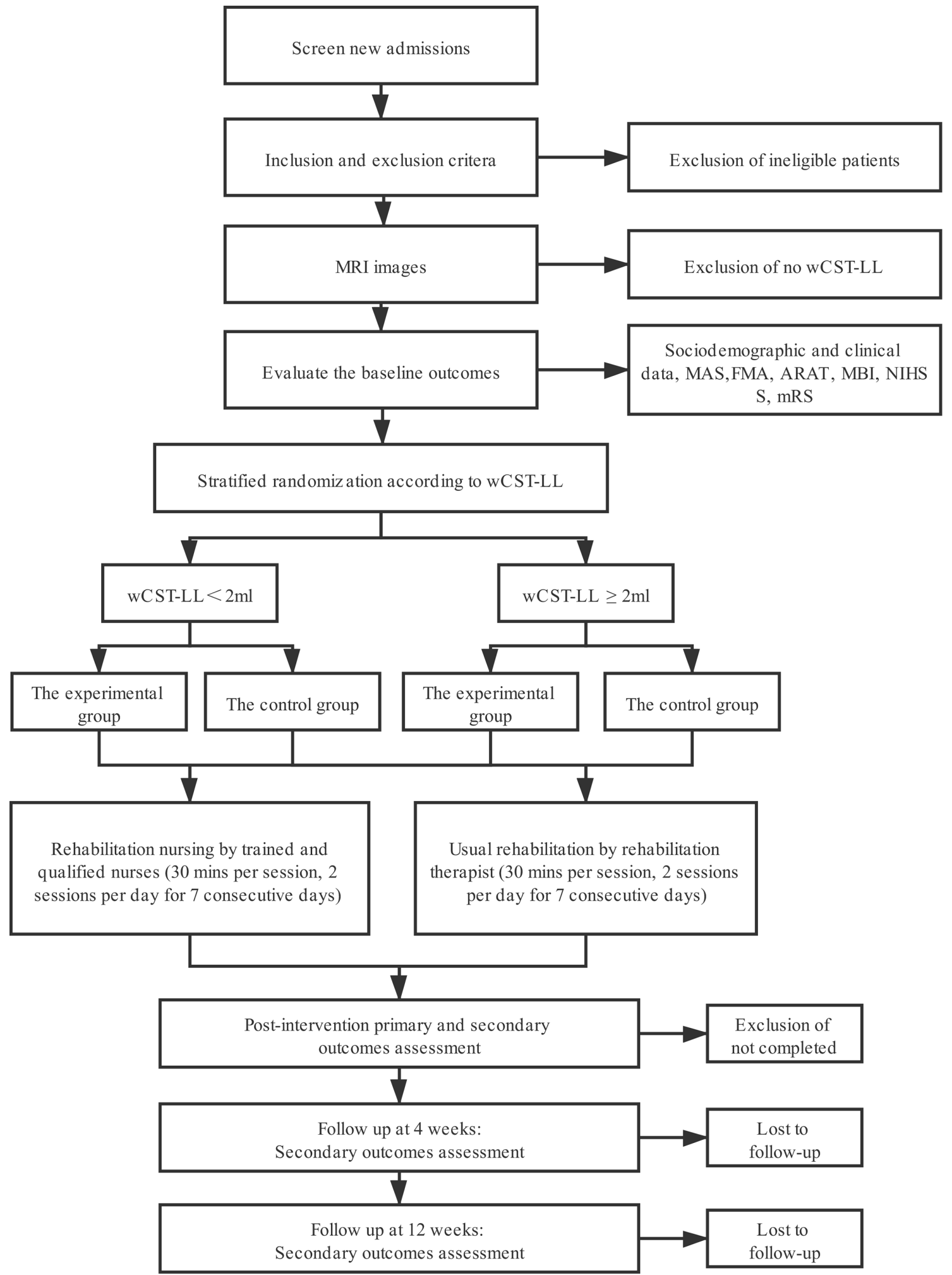

Figure 1 Flow diagram of study protocol. wCST-LL, weighted corticospinal tract lesion load; primary outcomes measures: MAS, Motor Assessment Scale; FMA, fugl-meyer assessment; ARAT, action research arm test; secondary outcomes measures: $\mathrm{MBI}$, modified Barthel Index; mRS, modified Rankin Scale; NIHSS: National Institute of Health Stroke Scale;

(30 min per session, two sessions per day, for seven consecutive days, in individual training programmes), while the control group will receive usual rehabilitation over the same time and frequency (figure 1). The protocol follows the Standard Protocol Items:
Recommendations for Interventional Trials (SPIRIT) guidelines $^{30}$ and fulfilment of the SPIRIT checklist (online supplemental file 1) and Template for Intervention Description and Replication (TIDieR) checklist (online supplemental file 2). 


\section{Subjects and setting}

The neurologist makes the diagnosis of acute ischaemic stroke confirmed by CT or MRI. The acute phase is defined as within 2 weeks after the onset of stroke, mild stroke within 1 week or severe stroke within 1 month. ${ }^{31}$ The study will be conducted at a tertiary hospital with one stroke unit and two neurology wards in southeastern China.

\section{Eligibility criteria}

Patients

The inclusion criteria for patients are (1) between 18 and 90 years old; (2) diagnosed as having an ischaemic stroke by CT or MRI and meeting the diagnostic criteria of WHO; (3) having an initial stroke within 7 days, with limb dysfunction (muscle strength is less than 5); (4) having no contraindications for MRI examination and the completed examination as having good image quality and complete clinical data; (5) maintaining consciousness (National Institute of Health Stroke Scale (NIHSS) consciousness level 0 or 1) and (6) having signed an informed consent form.

The exclusion criteria are having (1) blood vessels that were recanalised after thrombolysis; (2) cardiopulmonary dysfunction; a history of craniocerebral trauma, with fracture trauma or with rheumatoid arthritis or already had a physical disability or other diseases that had an impact on the affected limb; (3) cognitive impairment or other mental illness that prevents cooperation with researchers and (4) doctors considered unsuitable to participate in this study.

\section{Nurses}

The inclusion criteria for nurses are having (1) more than 5 years experience in neurology wards; (2) worked as a nurse team leader; (3) volunteered to participate in this study and (4) been trained and qualified through assessment of patient rehabilitation by a therapist team.

\section{Sample size}

Our pilot study showed that in patients with wCST-LL $<2 \mathrm{~mL}$, the mean scores of the total MotorAssessment Scale (MAS) of the intervention group and the control group after the intervention were 29.23 and 24.40 , and the SDs were 14.50 and 13.89 , respectively. The noninferiority threshold $\delta$ is determined by experts based on one-half of the SD of the control group, $\delta=6.94, \alpha=0.025$ (one tailed), $\beta=0.8$, The total sample size of the two groups is more than 134. The ratio between the wCST-LL $<2 \mathrm{~mL}$ and $\geq 2 \mathrm{~mL}$ is approximately $2: 1$; the minimum number required for the four groups is more than 201. Taking into consideration the dropout rate of about $10 \%$, the sample size is more than 224 .

\section{Recruitment}

According to our sample size, 224 patients with stroke will be recruited from our hospital for a period of 12-18 months. The assessors will screen newly admitted patients with a diagnosis of acute ischaemic stroke at the hospital electronic information service platform every day, using the inclusion and exclusion criteria, and the independent research coordinator will monitor the recruitment. Eligible patients will sign the written informed consent (online supplemental file 3). Recruitment status will be reported daily, recruitment progress will be discussed weekly and adjustments will be made accordingly. The study director will have access to the final results and can decide to terminate the trial.

\section{Randomisation and blinding}

After stratifying into large and small groups by wCST-LL, each group of patients will be randomly divided into an experimental group and a control group by a computergenerated random number method. After eligible patients sign an informed consent, a trained and qualified assessor, blinded for the implementation of the intervention, will conduct the baseline assessments. Patients will be assigned a number according to the order of baseline assessment, and each ordinal number will correspond to a random number. Due to the characteristics of the faceto-face treatment, patients may know their grouping and those who deliver the intervention cannot achieve blindness, thus, it is a single assessor-blinded study. Unblinding will be conducted, after all data are checked and data analysis is completed. Once the data are unblinded, no other statistical analyses will be conducted.

\section{Concealment of allocation}

An independent researcher coordination method, analogous to the centralised randomising system to group patients will be used to avoid selection bias. The randomisation is conducted by an independent research coordinator, who will check the baseline assessment ordinal number that corresponds to the random number and inform the therapists or head nurses but not the assessors about the allocation of the patient. The head nurse of each ward notifies the nurse responsible for the intervention delivery. The allocation table will be only visible to the independent research coordinator, who will not be involved in the enrolment, assessment or intervention process. Post-intervention assessments will be conducted when patients are available, to avoid knowledge about who delivers the rehabilitation, as a means to guarantee allocation concealment. Before the unblinding stage, assessors and statisticians will be fully blinded about the allocation details.

\section{Interventions}

\section{Experimental group}

The experimental group will use a rehabilitation nursing programme graded based on the MBI score. In this programme, the MBI is divided into five levels (the total score is 100 points, and each 20 points comprises a level). Each level provides some rehabilitation training items, including good limb position, Bobath therapy, passive movement, bridge movement, bed-roll exercises, sitting-up and standing-up training, sit-to-stand training, 
balance and transfer training, limb weight training, ADL training, and step and walking training. Nurses first assess the patient's MBI and then select the corresponding level of rehabilitation training items. The training programme is patient tailored and progressive, related to the patient's goals and caregiver's participation. It will be conducted twice a day, with $30 \mathrm{~min}$ for each session, for seven consecutive days, and may be adjusted to a different level based on the third-day post-intervention (ta) MBI score change. We will record the date, time, items and number of task repeats daily in the self-designed rehabilitation nursing record form as a means to improve adherence to protocols.

\section{Control group}

Patients in the two groups receive the same amount of basic nursing care. In addition, the control group will be given usual rehabilitation for function recovery by therapists (healthcare professionals who majored in rehabilitation medicine and have at least 3 years of work experience), based on the patient's condition and personal goals, exercises will involve physical therapy including: muscle strength training, joint mobility exercises, balance training, knee extension and ankle plantar flexion training, trunk control ability training, limb weight training; occupational therapy including: maintain normal posture, passive movement, active assistance to active training, ADL training and training of standing centre of gravity transfer. Some items are same with the experimental group, intervention time also is delivered same as experimental group (details see online supplemental file 4). Both group interventions will be conducted face-to-face at the patient's bedside or in another ward environment. Patients will be allowed to receive treatments deemed necessary by their attending physician with no interference as in the experimental group.

\section{Criteria for discontinuing or modifying the intervention in both groups}

Four criteria will be used for discontinuing or modifying the intervention in both groups: (1) patients with previously unknown severe cardiopulmonary disease after enrolment; (2) patients with progressive stroke or complications that precluded rehabilitation; (3) patients transferred to another hospital or other department or were reluctant to continue and (4) no CST damage was confirmed in the later MRI results. An effort will be made to collect data on these participants and to include their results in post-intervention analyses.

\section{Outcome measures}

Primary outcome measures

The MAS will be used to assess the patient's ability to perform functional activities rather than just cooperative motor patterns. The scale has eight areas of motor function, for which each item is scored from 0 to 6 ; the scale is highly reliable, with an inter-rater correlation of 0.95 and a test-retest correlation of $0.98 .^{32}$ The Spearman correlation coefficient between the total MAS score and the FMA after stroke reflects good validity $(r=0.96$, excluding the general tonus item) and scores range from 0 to $48 .^{33}$ This study focused on the overall function of patients, therefore, we choose the total score as the main outcome measures.

The FMA is the most widely accepted movement function scale in patients with stroke, with a reliability of at least $0.95 .^{34}$ The simplified FMA uses a 100 -point motor domain and has been demonstrated to have excellent reliability and construct validity. ${ }^{35}$ The simplified FMA has 50 evaluation items, divided into three levels $(0,1$ or 2 points). A total score of fewer than 50 points indicates that the patient has severe motor dysfunction; a score between 50 and 84 points indicates obvious motor dysfunction; a score between 85 and 95 is classified as moderate motor dysfunction; a score between 96 and 99 is classified as mild motor dysfunction.

Upper limb functioning will be assessed with the ARAT scale, which has 19 items divided into four-arm movement tests: grasp, grip, pinch and gross. Performance on each item is rated on an ordinal scale that ranges from 0 to 3 points. ${ }^{36}$ The Spearman's rank correlation coefficients was excellent with the FMA $(\mathrm{r}=0.77-0.87),{ }^{37}$ and intraclass correlation coefficients (ICCs) for inter-rater and intrarater reliability ranged from 0.92 to $0.97 .^{38}$

\section{Secondary outcome measures}

The seven-level, modified Rankin Scale (mRS) measures neurological recovery in patients after stroke ${ }^{39}$ and can distinguish effective from ineffective trials. Inter-observer variability, using pooled reliability, yield a weighted kappa of $0.90 .{ }^{40}$ Its concurrent validity is demonstrated by strong correlations with measures of infarct volumes as well as with the BI ( $r=-0.89)$ in acute stroke. ${ }^{41}$

The MBI will be used to assess the degree of patient independence. The MBI is a 10 -item, 100 total points scale, and each item has five levels, with each item weighted differently. It has been widely used in China and has good reliability (ICC $=0.866-0.997)$ and high criterion-related validity $(\mathrm{r}=0.816-1.000)$ with the $\mathrm{BI} .{ }^{42}$

The degree of neurological deficit will be assessed by the NIHSS. For all 11 parameters, a value of 0 is normal, and the top score is 42 points. ${ }^{43}$ The total score, determined by neurologists and trained nurses, has a high level of agreement $(\mathrm{ICC}=0.92-0.96){ }^{44}$

Except for NIHSS and mRS, for which lower scores indicate better function, all of the scales show that the patient's function is better if the scores are higher. All assessments will be performed by two blinded assessors (prioritize that the same assessor for one patient), who were trained and qualified before a formal assessment and testing for Cohen's kappa $(\kappa \geq 0.75)$.

\section{Data collection}

Blinded assessors will collect the clinical and sociodemographic data in a case report form and outcome data at baseline $(\mathrm{t} 0)$ and after the intervention ( $\mathrm{t} 1-\mathrm{t} 3)$. The 


\begin{tabular}{|l|c|c|c|c|c|c|c|c|}
\hline & \multicolumn{6}{|c|}{ Study Period } \\
\hline & Enrolment & Allocation & \multicolumn{3}{|c|}{ Intervention } & \multicolumn{3}{|c|}{$\begin{array}{c}\text { Post- } \\
\text { intervention }\end{array}$} \\
\hline Time point & $\mathrm{t}_{0}$ & $\mathrm{t}_{0}$ & $\mathrm{t}_{0}$ & $\mathrm{t}_{3}$ & $\mathrm{t}_{\mathrm{l}}$ & $\mathrm{t}_{\mathrm{d}}$ & $\mathrm{t}_{2}$ & $\mathrm{t}_{3}$ \\
\hline Enrolment & & & & & & & & \\
\hline Eligibility screen & $\mathrm{X}$ & & & & & & & \\
\hline Informed consent & $\mathrm{X}$ & & & & & & & \\
\hline Allocation & & $\mathrm{X}$ & & & & & & \\
\hline Intervention & & & & & & & & \\
\hline Experimental group & & & & & & & & \\
\hline Control group & & & & & & & & \\
\hline Assessments & & & & & & & & \\
\hline $\begin{array}{l}\text { Clinical and } \\
\text { sociodemographic } \\
\text { data }\end{array}$ & & & $\mathrm{X}$ & & & & & \\
\hline $\begin{array}{l}\text { Motor Assessment } \\
\text { Scale }\end{array}$ & & & $\mathrm{X}$ & & $\mathrm{X}$ & & & \\
\hline $\begin{array}{l}\text { Fugl-Meyer } \\
\text { Assessment }\end{array}$ & & & $\mathrm{X}$ & & $\mathrm{X}$ & & & \\
\hline $\begin{array}{l}\text { Action Research Arm } \\
\text { Test }\end{array}$ & & & $\mathrm{X}$ & & $\mathrm{X}$ & & & \\
\hline $\begin{array}{l}\text { Modified Rankin } \\
\text { Scale }\end{array}$ & & & $\mathrm{X}$ & & $\mathrm{X}$ & & $\mathrm{X}$ & $\mathrm{X}$ \\
\hline $\begin{array}{l}\text { Modified Barthel } \\
\text { Index }\end{array}$ & & $\mathrm{X}$ & $\mathrm{X}$ & $\mathrm{X}$ & & $\mathrm{X}$ & $\mathrm{X}$ \\
\hline $\begin{array}{l}\text { National Institute of } \\
\text { Health Stroke Scale }\end{array}$ & & & $\mathrm{X}$ & & $\mathrm{X}$ & $\mathrm{X}$ \\
\hline
\end{tabular}

Figure 2 Schedule of enrolment, intervention and assessments. -t0: admitted but not grouped; t0: baseline, day of enrolment; ta: 3 days post-intervention, reevaluated Modified Barthel Index to adjust protocol; 1 1: 7 days post-intervention; td: on the day of discharge; t2: 4 weeks post-intervention; t3: 12 weeks post-intervention.

independent research coordinator will check the assessment results before the intervention and modify any inconsistencies. All data will be entered into an Excel spreadsheet by one researcher and checked by another before randomisation, baseline assessment will be completed to avoid delaying the implementation of the intervention process. The schedule of enrolment, intervention and assessments is presented in figure 2.

\section{Data monitoring}

Patients will be strictly observed during the intervention, and all wards are to report any adverse events that occur from the baseline on. If serious complications, such as falls or health-threatening conditions, are identified, the study will be terminated immediately. The cause of the complication will be investigated, and feedback will be provided to the data monitoring committee, which comprises researchers who are independent of the sponsor and the quality control committee of the hospital. Intervention compliance or fidelity is evaluated by the data-monitoring committee, and daily work progress reporting strategies are used to maintain fidelity.

\section{Statistical methods}

As noted, 224 patients are needed to achieve noninferiority at a one-tailed $\alpha$-value of 0.025 . Our descriptive statistics will include means and SDs for continuous variables and numbers and proportions for categorical variables. We will conduct a non-inferiority test between the intervention and control groups for the primary and secondary outcomes without regard to the wCST-LL. Then compare the differences between the intervention and control groups in wCST-LL $<2 \mathrm{~mL}$ and wCST-LL $>2 \mathrm{~mL}$ group, respectively.

Between-group differences at baseline will be studied using Mann-Whitney U tests, Student's t-tests, or Fisher's exact tests. Covariance analysis will be used to adjust for some confounding factors, the post hoc analysis will use per-protocol analysis, and the interaction between group and time will be tested, with a mixed effect model as implemented, if possible. All analyses will be conducted using IBM SPSS Statistics, V.22.0 (IBM).

\section{Patient and public involvement statement}

During the trial, the patient needs to complete some rehabilitation exercises according to the instructions, and the family caregiver needs to be educated to assist in the exercises. Patients and family caregivers have the right to choose exercise items and to give feedback. There are no plans to disseminate the study results to participants.

\section{Ethics and dissemination}

The Human Research Ethics Committee of the second affiliated hospital of Zhejiang University School of Medicine has approved this study. The study dissemination will be published in a peer-reviewed journal and/or presented at national or international conferences. 


\section{DISCUSSION}

Our study may provide some evidence that rehabilitation nursing is not inferior to usual rehabilitation. The milestone research, A Very Early Rehabilitation Trial after stroke, showed that complex interventions in stroke care are possible and can be led by physiotherapists and nurses. ${ }^{45}$ A large observational study found that, for every $1 \%$ increase in the number of certified nurses per unit, the length of stay was reduced by about $6 \%$, indicating an added value for nurses with this expertise. ${ }^{46}$ Nevertheless, there is a limited perception of nursing practice and very little research on nurses' role in poststroke rehabilitation. ${ }^{47} \mathrm{~A}$ review shows that the role of certain nursing interventions is still ambiguous, and the claim that nurses are 'rehabilitators par excellence' has not yet been fully realised. ${ }^{49}$ The lack of studies on the nature of nursing interventions ${ }^{49}$ makes our research even more valuable. Nursing can improve the neurological function and ADLs of stroke patients. ${ }^{20}$ Nurses were able to translate the development of disparate physical skills into ADLs. Thus the MBI-based rehabilitation nursing make full use of their role to develop new partnerships with patients beyond the usual rehabilitation by the therapists. With the arrival of an ageing society, rehabilitation services need to be strengthened to achieve the 2030 sustainable development goal. $^{50}$ Thus, research on rehabilitation nursing is needed to clarify and develop opportunities for rehabilitation registered nurses. ${ }^{51}$

Nevertheless, a rehabilitation nursing programme with a task oriented is consistent with stroke guidelines and the principles of holistic nursing, but is different from most current studies. $^{2752}$ In our study, patients are divided into large and small groups based on wCST-LL, and rehabilitation nursing is implemented to determine the effects of wCST-LL on rehabilitation outcomes, which have not been well studied.

This protocol also has some limitations. First, the time for rehabilitation intervention is 7 days, which is relatively short, as it is limited by the average length of stay of patients with acute stroke in a research hospital. This average length of stay is longer than that in the USA, for which the median length of stay for patients with ischaemic stroke is just 4 days. ${ }^{51}$ Follow-up will occur at 4 and 12 weeks, as this is a preliminary study of our programme, notably, the recovery of stroke motor function is faster in the first 4 months and may not change much later. ${ }^{53}$ Besides, this is a single-site RCT and, thus, the findings may not apply to other populations. Future research should involve a multicentre study to confirm and to extend the results of this study.

In this study, we hypothesised that rehabilitation nursing might have a non-inferiority effect with usual rehabilitation for the motor function recovery for patients with acute ischaemic stroke. We expect that this trial will provide evidence that it is effective to provide rehabilitation nursing during the acute inpatient phase of stroke.
Acknowledgements The authors would like to thank all of the patients and their relatives who are involved in this study. We also appreciate the multidisciplinary teamwork of the neurology department, rehabilitation department and radiology department.

Contributors JW drafted the present manuscript, collects the data, and is responsible for the integrity of the data and the accuracy of the analysis. YC contributed to the development of the protocol and is the independent research coordinator in the study. YZ revised the final draft of the manuscript and contributed to the development and carrying out of the protocol. ML collects the data and contributed to help modify the protocol. JJ contributed to the design and procurement of funding and the approval for this study and is the director responsible for the whole study. All authors read and approved the final manuscript.

Funding This study was supported by the National Natural Science Foundation of China (no: 81871839).

Disclaimer This agency did not play a role in study design or implementation, statistical analysis, or the writing of the paper.

Competing interests None declared.

Patient and public involvement Patients and/or the public were not involved in the design, or conduct, or reporting, or dissemination plans of this research.

Patient consent for publication Not required.

Provenance and peer review Not commissioned; externally peer reviewed.

Open access This is an open access article distributed in accordance with the Creative Commons Attribution Non Commercial (CC BY-NC 4.0) license, which permits others to distribute, remix, adapt, build upon this work non-commercially, and license their derivative works on different terms, provided the original work is properly cited, appropriate credit is given, any changes made indicated, and the use is non-commercial. See: http://creativecommons.org/licenses/by-nc/4.0/.

ORCID iD

Jianmiao Wang http://orcid.org/0000-0002-8371-517X

\section{REFERENCES}

1 World Health Organization. Global health estimates 2016: disease burden by cause, age, sex, by country and by region, 2000-2016. Geneva: World Health Organization, 2018.

2 Wang W, Jiang B, Sun $\mathrm{H}$, et al. Prevalence, incidence, and mortality of stroke in China: results from a nationwide population-based survey of 480687 adults. Circulation 2017;135:759-71.

3 Lai C-L, Tsai M-M, Luo J-Y, et al. Post-acute care for stroke - a retrospective cohort study in Taiwan. Patient Prefer Adher 2017;11:1309-15.

4 Hatem SM, Saussez G, Della Faille M, et al. Rehabilitation of motor function after stroke: a multiple systematic review focused on techniques to stimulate upper extremity recovery. Front Hum Neurosci 2016;10:442-42.

5 GBD 2015 Disease and Injury Incidence and Prevalence Collaborators. Global, regional, and national incidence, prevalence, and years lived with disability for 310 diseases and injuries, 19902015: a systematic analysis for the Global Burden of Disease study 2015. Lancet 2016;388:1545-602.

6 Duncan PW, Zorowitz R, Bates B, et al. Management of adult stroke rehabilitation care: a clinical practice guideline. Stroke 2005;36:e100-43.

7 Zhang WW, Speare S, Churilov L, et al. Stroke rehabilitation in China: a systematic review and meta-analysis. Int J Stroke 2014;9:494-502.

8 Chen C-M, Lee M, Yang Y-H, et al. Association between clinical and laboratory markers and 5-year mortality among patients with stroke. Sci Rep 2019;9:11521.

9 Chen C-M, Yang Y-H, Chang C-H, et al. Effects of transferring to the rehabilitation ward on long-term mortality rate of first-time stroke survivors: a population-based study. Arch Phys Med Rehabil 2017;98:2399-407.

10 Fan Y-T, Lin K-C, Liu H-L, et al. Changes in structural integrity are correlated with motor and functional recovery after post-stroke rehabilitation. Restor Neurol Neurosci 2015;33:835-44.

11 Song J, Nair VA, Young BM, et al. DTI measures track and predict motor function outcomes in stroke rehabilitation utilizing $\mathrm{BCI}$ technology. Front Hum Neurosci 2015;9:195.

12 Jin J-F, Guo Z-T, Zhang Y-P, et al. Prediction of motor recovery after ischemic stroke using diffusion tensor imaging: a meta-analysis. World J Emerg Med 2017;8:99-105. 
13 Feng W, Wang J, Chhatbar PY, et al. Corticospinal tract lesion load: an imaging biomarker for stroke motor outcomes. Ann Neurol 2015;78:860-70.

14 Powers William J, Rabinstein Alejandro A, Ackerson T, et al. 2018 Guidelines for the early management of patients with acute ischemic stroke: a guideline for healthcare professionals from the American Heart Association/American Stroke Association. Stroke 2018;49:e46-99.

15 Bettger JP, Li Z, Xian Y, et al. Assessment and provision of rehabilitation among patients hospitalized with acute ischemic stroke in China: findings from the China National Stroke Registry II. Int $J$ Stroke 2017;12:254-63.

16 Asakawa T, Zong L, Wang L, et al. Unmet challenges for rehabilitation after stroke in China. Lancet 2017;390:121-2.

$17 \mathrm{Li} \mathrm{Z}$, Jiang Y, Li H, et al. China's response to the rising stroke burden. BMJ 2019;364.

18 Scrutinio D, Giardini A, Chiovato L, et al. The new frontiers of rehabilitation medicine in people with chronic disabling illnesses. Eur $J$ Intern Med 2019;61:1-8.

19 Whitehead S, Baalbergen E. Post-stroke rehabilitation. S Afr Med J 2019;109:81-3.

20 Guan YX, Guo N, Gao H, et al. Study on application of continuous nursing in rehabilitation period of stroke patients. Acta Medica Mediterr 2019;35:539-43.

21 Abd El-Hay SA, Abed Allah AK, Tag El Din ESA. Effect of implementing designed educational training program for neurological nurses on clinical outcomes of stroke patients. Clin Nurs Stud 2018;6:121.

22 Burton CR. A description of the nursing role in stroke rehabilitation. $J$ Adv Nurs 2000;32:174-81.

23 Woon C. Nursing at the centre of stroke recovery in the acute setting: prioritising early rehabilitation. British $J$ Neurosci Nurs 2016;12:23-8.

24 Clarke DJ. Nursing practice in stroke rehabilitation: systematic review and meta-ethnography. J Clin Nurs 2014;23:1201-26.

25 Theofanidis D, Gibbon B. Nursing interventions in stroke care delivery: an evidence-based clinical review. J Vasc Nurs 2016;34:144-51.

26 Duncan PW, Sullivan KJ, Behrman AL, et al. Body-weightsupported treadmill rehabilitation after stroke. N Engl J Med 2011;364:2026-36.

27 Wolf SL, Winstein CJ, Miller JP, et al. Effect of constraintinduced movement therapy on upper extremity function 3 to 9 months after stroke: the EXCITE randomized clinical trial. JAMA 2006;296:2095-104.

28 Hubbard IJ, Parsons MW, Neilson C, et al. Task-specific training: evidence for and translation to clinical practice. Occup Ther Int 2009;16:175-89.

29 American Occupational Therapy Association. Occupational therapy practice framework: domain and process (3rd edition). Am J Occup Ther 2014;68:S1-48.

30 Chan A-W, Tetzlaff JM, Gøtzsche PC, et al. SPIRIT 2013 explanation and elaboration: guidance for protocols of clinical trials. BMJ 2013;346:e7586.

31 Chinese Society of Neurology. Society of cerebrovascular disease. Chinese guidelines for diagnosis and treatment of acute ischemic stroke 2018. Chin J Neurol 2018;51:666-82.

32 Carr JH, Shepherd RB, Nordholm L, et al. Investigation of a new motor assessment scale for stroke patients. Phys Ther 1985;65:175-80.
33 Malouin F, Pichard L, Bonneau C, et al. Evaluating motor recovery early after stroke: comparison of the Fugl-Meyer assessment and the motor assessment scale. Arch Phys Med Rehabil 1994;75:1206-12.

34 Hsieh Y-W, Hsueh I-P, Chou Y-T, et al. Development and validation of a short form of the Fugl-Meyer motor scale in patients with stroke. Stroke 2007;38:3052-4.

35 Gladstone DJ, Danells CJ, Black SE. The Fugl-Meyer assessment of motor recovery after stroke: a critical review of its measurement properties. Neurorehabil Neural Repair 2002;16:232-40.

36 Hsieh CL, Hsueh IP, Chiang FM, et al. Inter-Rater reliability and validity of the action research arm test in stroke patients. Age Ageing 1998;27:107-13.

37 Rabadi MH, Rabadi FM. Comparison of the action research arm test and the Fugl-Meyer assessment as measures of upper-extremity motor weakness after stroke. Arch Phys Med Rehabil 2006;87:962-6.

38 Nijland R, van Wegen E, Verbunt J, et al. A comparison of two validated tests for upper limb function after stroke: the Wolf motor function test and the action research arm test. $J$ Rehabil Med 2010;42:694-6.

39 van Swieten JC, Koudstaal PJ, Visser MC, et al. Interobserver agreement for the assessment of handicap in stroke patients. Stroke 1988;19:604-7

40 Broderick JP, Adeoye O, Elm J. Evolution of the modified Rankin scale and its use in future stroke trials. Stroke 2017;48:2007-12.

41 Kwon S, Hartzema AG, Duncan PW, et al. Disability measures in stroke. Stroke 2004;35:918-23.

42 Min Y, Yuanyuan WU, Yan T. Validity and reliability of the simplified Chinese version of modified Barthel index for Chinese stroke patients. Chin J Phys Med Rehabil 2008;3:185-8.

43 Kwah LK, Diong J. National Institutes of health stroke scale (NIHSS). $J$ Physiother 2014;60:61.

44 Dewey HM, Donnan GA, Freeman EJ, et al. Interrater reliability of the National Institutes of health stroke scale: rating by neurologists and nurses in a community-based stroke incidence study. Cerebrovasc Dis 1999;9:323-7.

45 Rothwell PM. AVERT: a major milestone in stroke research. Lancet 2015;386:7-9.

46 Nelson A, Powell-Cope G, Palacios P, et al. Nurse staffing and patient outcomes in inpatient rehabilitation settings. Rehabil Nurs 2007;32:179-202.

47 Zerna C, Burley T, Green TL, et al. Comprehensive assessment of disability post-stroke using the newly developed miFUNCTION scale. Int J Stroke 2020;15:167-74.

48 Aadal L, Angel S, Dreyer P, et al. Nursing roles and functions in the inpatient neurorehabilitation of stroke patients: a literature review. $J$ Neurosci Nurs 2013;45:158-70.

49 O'Connor SE. Nursing and rehabilitation: the interventions of nurses in stroke patient care. J Clin Nurs 1993:2:29-34.

50 Gimigliano F, Negrini S. The World Health Organization "rehabilitation 2030: a call for action". Eur J Phys Rehabil Med 2017:53:155-68.

51 Winstein CJ, Stein J, Arena R, et al. Guidelines for adult stroke rehabilitation and recovery: a guideline for healthcare professionals from the American heart association/American stroke association. Stroke 2016;47:e98-169.

52 Taub E, Uswatte G, Pidikiti R. Constraint-induced movement therapy: a new family of techniques with broad application to physical rehabilitation--a clinical review. J Rehabil Res Dev 1999;36:237-51.

53 Winstein CJ, Wolf SL, Dromerick AW, et al. Effect of a task-oriented rehabilitation program on upper extremity recovery following motor stroke: the ICARE randomized clinical trial. JAMA 2016;315:571-81. 